\title{
Segment Training Based Individual Channel Estimation for Multi-pair Two-Way Relay Network with Power Allocation
}

\author{
Xiandeng $\mathrm{He}^{1}$, Ronghua Zhou ${ }^{1}$, Nan Chen ${ }^{1}$ and Shun Zhang ${ }^{1}$ \\ ${ }^{1}$ State Key Laboratory of Integrated Service Networks, Xi'dian University \\ Xi'an, China \\ [e-mail: xdhe@mail.xidian.edu.cn; rhzhou@stu.xidian.edu.cn; \\ nchen@mail.xidian.edu.cn; zhangshunsdu@gmail.com] \\ *Corresponding author: Xiandeng He
}

Received May 22, 2017; revised September 7, 2017; accepted September 19, 2017;

published February 28, 2018

\begin{abstract}
In this paper, we design a segment training based individual channel estimation (STICE) scheme for the classical two-way relay network (TWRN) with multi-pair sources (MPS) and amplify-and-forward (AF). We adopt the linear minimum mean square error (LMMSE) channel estimator to minimize the mean square error (MSE) without channel estimation error, where the optimal power allocation strategy from the relay for different sources is obtained. Then the MSE gains are given with different source pairs among the proposed power allocation scheme and the existing power allocation schemes. Numerical results show that the proposed method outperforms the existing ones.
\end{abstract}

Keywords: Channel estimation, LMMSE, power allocation, two-way relay networks, amplify-and-forward

Project supported by the National Natural Science Foundation of China (Nos. 61372076 and 61301171), the 111 Project (No. B08038), and the Fundamental Research Funds for the Central Universities (Nos. K5051201022).

We express our thanks to the associate editor and the reviewers for the time and effort they have put into our paper. 


\section{Introduction}

The two-way relay network (TWRN) has been popular studied due to its capability of improving the spectral efficiency compared to the one-way relay network (OWRN) [1-4]. Several simple but effective relay protocols, such as LMMSE transceiver [5][6], beamforming [7][8], distributed space-time coding (DSTC) [9][10], linear precoding [11], etc., have been proposed to capture the advantages for the relay networks.

Following the same guideline, the training design and power allocation for amplify-and-forward (AF) based TWRN have been studied in [12-16]. In [12], the author considered a two-hop non-regenerative multiple-input multiple-output (MIMO) relay system and computed the optimal source and relay pilot matrixes by using LMMSE which allows to use the prior knowledge of channel correlations. To make the method more practical, the optimal training and optimal relay's power allocation were given resorting to LMMSE estimators in our previous work [4] and [6]. A new channel estimation prototype was proposed in [14] to allow the relay firstly estimate the channel parameter and then allocate the powers based on these parameters. Both [15] and [16] studied in two-way MIMO relay networks, where they derived the optimal training structure and the optimal power allocation to minimize MSE. [17] extended the channel estimation to frequency selective channels by applying the orthogonal frequency division multiplex (OFDM) modulation.

All researchs above, including our previous work, are mainly focused on the classical three-node relay network where two sources exchange their information via a single relay node. However, in real systems, one relay may work for multi-pair sources, which has been examined in $[18,19]$. The authors in $[18,19]$ focused on the estimation of the convolution channels (called co-channels here) between the source-to-relay links and relay-to-source ones. However, purely knowing the co-channels is insufficient to support the optimal system design in certain scenarios. For example, in the relay beamforming scheme, the individual channels (called in-channels here) between the sources and the relays are required to design the relay's operation and to let the source predict the relay's operation[7, 8].

In this paper, we propose a practical segment training based in-channel estimation(STICE) algorithm under the multi-pair sources(MPS) TWRN scenario. Furthermore, to avoid the interference, we adopt orthogonal frequency division multiple access(OFDMA) and schedule source paris into different subcarriers. Then, we derive the MSEs of the in-channel estimations, and derive the optimal power allocation for different sources.

The paper is organized as follows. Section 2 presents the system model. In Section 3, we derive the expressions of the power allocation factors for different sources. Simulation results are provided in Section 4. Finally, Conclusions are made in Section 5.

\section{System Model}

A typical $2 n+1$-node TWRN with $n$ pairs of sources $\mathbf{s}_{a}=\left[s_{a 1}, s_{a 2}, \cdots, s_{a n}\right]^{T}$, $\mathbf{s}_{b}=\left[s_{b 1}, s_{b 2}, \cdots, s_{b n}\right]^{T}$ and one relay node $R$ is shown in Fig. 1. Each node works in half-duplex mode, and FDD is applied for different source pairs. We assume that there is no direct link between $\mathbf{s}_{a}$ and $\mathbf{s}_{b}$. The in-channels from sources to relay and that from relay to sources are reciprocal, and assumed to be block fading. The in-channels between $S_{a i}$ and $R, R$ 
and $\boldsymbol{s}_{\boldsymbol{b} \boldsymbol{i}}$ are denoted by $\boldsymbol{h}_{\boldsymbol{i}}$ and $\boldsymbol{g}_{\boldsymbol{i}}, i=1,2, \ldots, n$, respectively. We assume that all $\boldsymbol{h}_{\boldsymbol{i}}$ and $\boldsymbol{g}_{\boldsymbol{i}}$ are zero mean circularly symmetric complex Gaussian (CSCG) random variables, i.e., $h_{i} \sim C N\left(0, \sigma_{h_{i}}^{2}\right)$ and $g_{i} \sim C N\left(0, \sigma_{g_{i}}^{2}\right)$. Furthermore, $h_{i}$ and $g_{i}$ are assumed to be independent, and perfect synchronization are among all these nodes.

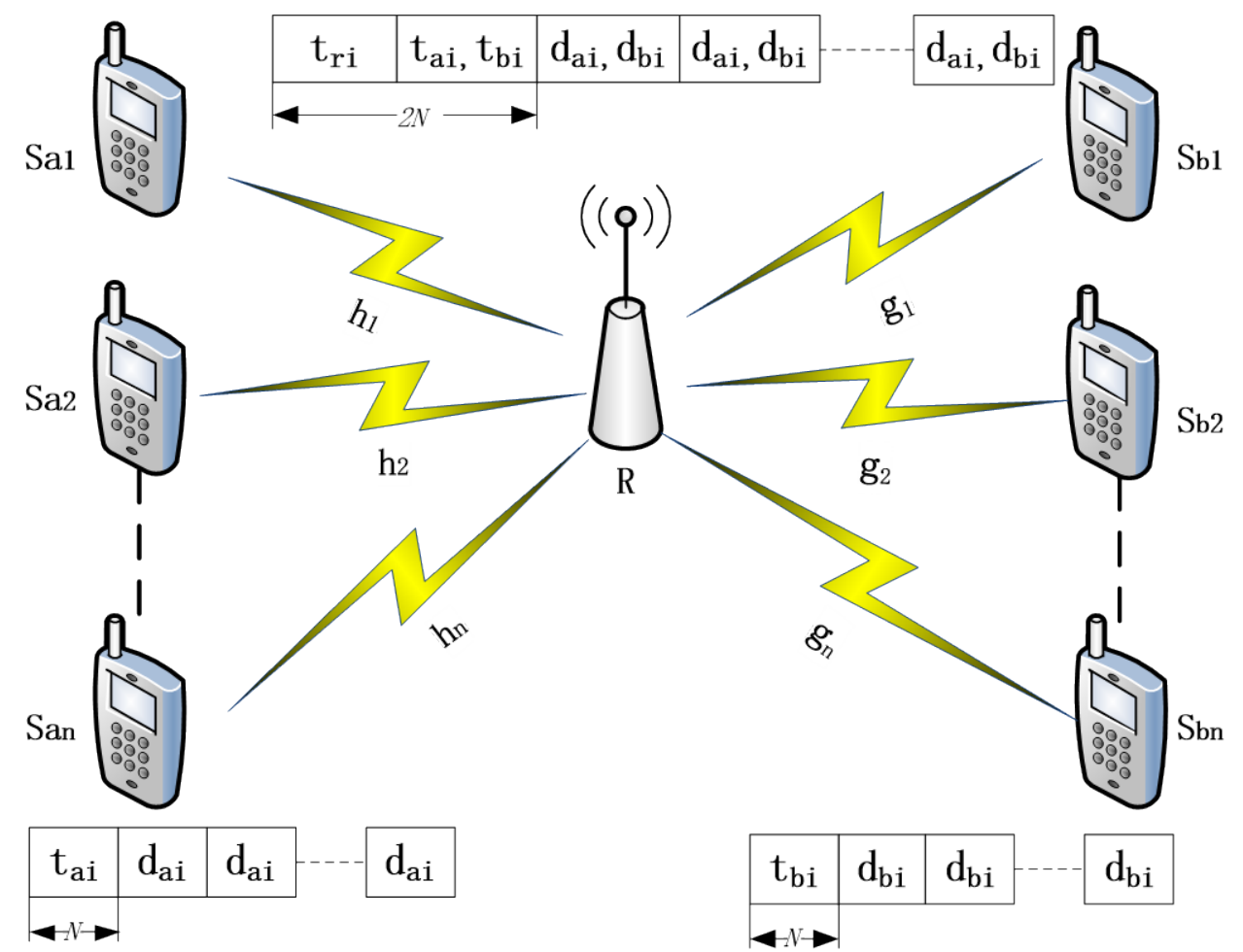

Fig. 1. The STICE scheme for the classical 2n+1-node TWRN

As shown in Fig. 1, during the STICE, one round of the signal exchange between $\mathbf{s}_{\boldsymbol{a}}$ and $\mathbf{s}_{b}$ can be divided into two phases. Phase I, $\boldsymbol{s}_{\boldsymbol{a} i}$ and $\boldsymbol{s}_{\boldsymbol{b} i}$ concurrently send one signal frame, including one source-training block and several data blocks, to $R$, and the source-training block and data blocks from $s_{j i}, j=a, b$, are denoted by the column vectors $\mathbf{t}_{j i}$ and $\mathbf{d}_{j i}$, respectively; Phase II, $R$ imposes one relay-training block $\mathbf{t}_{r i}$ in front of the received signal frame, and broadcasts the coded frame to $s_{a i}$ and $s_{b i}$.

Without loss of generality, all the training blocks, i.e., $\mathbf{t}_{a i}, \mathbf{t}_{b i}$ and $\mathbf{t}_{r i}$, are assumed to have the same length $N$. The received training block at $R$ in phase I can be expressed as

$$
\mathbf{X}_{r}=\mathbf{T}_{a} \mathbf{h}+\mathbf{T}_{b} \mathbf{g}+\mathbf{N}_{r}
$$

in which $\mathbf{T}_{a}=\left[\mathbf{t}_{a 1}, \mathbf{t}_{a 2}, \ldots \mathbf{t}_{a n}\right], \mathbf{T}_{b}=\left[\mathbf{t}_{b 1}, \mathbf{t}_{b 2}, \ldots \mathbf{t}_{b n}\right], \mathbf{h}=\operatorname{diag}\left\{h_{i}\right\}, \mathbf{g}=\operatorname{diag}\left\{g_{i}\right\}$, and 
$\mathbf{N}_{r}=\left[\mathbf{n}_{r 1}, \mathbf{n}_{r 2}, \ldots \mathbf{n}_{r n}\right]$ represents the additive-white Gaussian noise (AWGN) vector where each element is an $N \times 1$ noise vector with variance $N \sigma_{n}^{2}$. The power of $\mathbf{T}_{j}$ is denoted by $\mathbf{P}_{j}=\mathbf{T}_{j}^{H} \mathbf{T}_{j}=\operatorname{diag}\left\{P_{j i}\right\}, j=a, b, i=1,2, \ldots, n$.

In the phase II, the relay-training $\mathbf{t}_{r i}$ is imposed in front of the received source-training at $R$, and the overall training broadcasted by $R$ can be written as the $2 N \times n$ vector

$$
\mathbf{R}_{t}=\left[\mathbf{T}_{r}^{T},\left(\mathbf{A} \mathbf{X}_{r}\right)^{T}\right]^{T},
$$

where $\mathbf{T}_{r}=\left[\mathbf{t}_{r 1}, \mathbf{t}_{r 2}, \ldots \mathbf{t}_{r n}\right]$, the factor $\mathbf{A}=\operatorname{diag}\left\{\alpha_{i}\right\}$ controls the power allocated to the forwarded relay-training part.

The powers of $\mathbf{T}_{r}$ and $\mathbf{R}_{t}$ are defined as $\mathbf{P}_{r}=\mathbf{T}_{r}^{H} \mathbf{T}_{r}$ and $\mathbf{P}_{t}=\mathbf{R}_{t}^{H} \mathbf{R}_{t}=\operatorname{diag}\left\{P_{t i}\right\}$, respectively. The total relay power can be given by $P_{t}=\sum_{\mathrm{i}=1}^{\mathrm{n}} P_{t i}=\sum_{\mathrm{i}=1}^{\mathrm{n}} \beta_{i} P_{t}$, where $\beta_{i}$ controls the relay power allocated to the $i^{\text {th }}$ sources. Thus, from (2), we can obtain

$$
\mathbf{P}_{r}=\mathbf{P}_{t}-\mathbf{A}^{2}\left(\mathbf{C}_{\mathbf{h}} \mathbf{P}_{a}+\mathbf{C}_{\mathbf{g}} \mathbf{P}_{b}+N \sigma_{n}^{2} \mathbf{I}\right),
$$

where $\mathbf{C}_{\mathbf{h}}=E\left\{\mathbf{h h}^{H}\right\}, \mathbf{C}_{\mathbf{g}}=E\left\{\mathbf{g g}^{H}\right\}, \alpha_{i} \in\left[0, \sqrt{\frac{\beta_{i} P_{t}}{\sigma_{h i}^{2} P_{a i}+\sigma_{g i}^{2} P_{b i}+N \sigma_{n}^{2}}}\right]$ to make $P_{r i}$ a non-negative value. The received training at $\mathbf{s}_{a}$ can be divided into two parts, i.e.,

$$
\begin{gathered}
\mathbf{Z}_{a}^{r}=\mathbf{T}_{r} \mathbf{h}+\mathbf{N}_{a}^{r}, \\
\mathbf{Z}_{a}^{s}=\mathbf{T}_{a} \mathbf{A} \mathbf{h}^{2}+\mathbf{T}_{b} \mathbf{A h g}+\mathbf{N}_{r} \mathbf{A h}+\mathbf{N}_{a}^{s},
\end{gathered}
$$

where $\mathbf{N}_{a}^{r}=\left[\mathbf{n}_{a 1}^{r}, \mathbf{n}_{a 2}^{r}, \ldots, \mathbf{n}_{a n}^{r}\right]$ and $\mathbf{N}_{a}^{s}=\left[\mathbf{n}_{a 1}^{s}, \mathbf{n}_{a 2}^{s}, \ldots, \mathbf{n}_{a n}^{s}\right]$ are $N \times n$ AWGN matrixes at $\mathbf{s}_{a}$, each element is an $N \times 1$ noise vector with variance $N \sigma_{n}^{2}$.

\section{Segment Training Based In-channel Estimation}

When the number of source pair is 1 , which is $n=1$, the model reduces to the classical three-node TWRN where two sources exchange their information via a single relay node. This model has been carefully examined in our previous work [6], where we have derived and given the optimal power allocation factor $\alpha_{1}$ by Eqn. (22). When $\mathrm{n}>1$, the power allocation process can be divided into two steps: the allocation among different source pairs and that between source and relay. With respect to the former step, the power allocation is characterized by the parameter $\beta_{i}$, while the latter one is described by the parameter $\alpha_{i}$. If the factor $\beta_{i}$ is 
determined, according to the Eqn. (22) in [6], the optimal power allocation factor $\alpha_{i}$ can be given by

$$
\alpha_{o p t_{-} i}^{2}=\frac{F_{c}}{F_{b}}\left(1-\sqrt{1-\frac{F_{b} \beta_{i} P_{t}}{F_{a} F_{c}}}\right),
$$

where $F_{a}=\sigma_{h}^{2} P_{a}+\sigma_{g}^{2} P_{b}+N \sigma_{n}^{2}, F_{b}=(N-1) \sigma_{h}^{2} \sigma_{n}^{2}+\sigma_{h}^{4} P_{a}, F_{c}=\sigma_{h}^{2} \beta_{i} P_{t}+\sigma_{n}^{2}$.

From (4), we can formulate the standard LMMSE estimator of $\mathbf{h}$ as

$$
\hat{\mathbf{h}}=\mathbf{C}_{\mathbf{h}} \mathbf{T}_{r}^{H}\left(\sigma_{n}^{2} \mathbf{I}+\mathbf{T}_{r} \mathbf{C}_{\mathbf{h}} \mathbf{T}_{r}^{H}\right)^{-1} \mathbf{Z}_{a}^{r}
$$

The variances of both the error $\mathbf{e}_{\mathbf{h}}=\mathbf{h}-\hat{\mathbf{h}}$ and the estimated result $\hat{\mathbf{h}}$ are computed by

$$
\begin{gathered}
\mathbf{C}_{\mathbf{e}_{\mathbf{h}}}=\left(\mathbf{C}_{\mathbf{h}}^{-1}+\mathbf{P}_{\mathbf{r}} \sigma_{n}^{-2}\right)^{-1}, \\
\mathbf{C}_{\hat{\mathbf{h}}}=\mathbf{C}_{\mathbf{h}}-\mathbf{C}_{\mathbf{e}_{\mathbf{h}}}=\left(\sigma_{n}^{2} \mathbf{I}+\mathbf{P}_{r} \mathbf{C}_{\mathbf{h}}\right)^{-1} \mathbf{P}_{r} \mathbf{C}_{\mathbf{h}}^{2},
\end{gathered}
$$

then $\mathbf{Z}_{a}^{s}$ in (5), can be rewritten as

$$
\mathbf{Z}_{a}^{s}-\mathbf{T}_{a} \mathbf{A} \hat{\mathbf{h}}^{2}=\mathbf{T}_{b} \mathbf{A} \hat{\mathbf{h}} \mathbf{g}+\mathbf{N}_{t},
$$

where $\mathbf{N}_{t}=2 \mathbf{T}_{a} \mathbf{A} \hat{\mathbf{h}} \mathbf{e}_{\mathbf{h}}+\mathbf{T}_{a} \mathbf{A} \mathbf{e}_{\mathbf{h}}{ }^{2}+\mathbf{T}_{b} \mathbf{A} \mathbf{e}_{\mathbf{h}} \mathbf{g}+\mathbf{N}_{r} \mathbf{A}\left(\hat{\mathbf{h}}+\mathbf{e}_{\mathbf{h}}\right)+\mathbf{N}_{a}^{s}$ is the equivalent noise at $\mathbf{s}_{a}$. Since the terms $\mathbf{e}_{\mathbf{h}}, \mathbf{g}, \mathbf{N}_{r}, \mathbf{N}_{a}^{s}$ are uncorrelated, the covariance matrix of $\mathbf{N}_{t}$ conditioned on $\hat{\mathbf{h}}$ can be derived as

$$
\mathbf{C}_{\mathbf{N}_{t} \hat{\mathbf{h}}}=E\left\{\mathbf{N}_{t} \mathbf{N}_{t}^{H}\right\}=\mathbf{T}_{a} \boldsymbol{\Psi}_{a} \mathbf{T}_{a}^{H}+\mathbf{T}_{b} \mathbf{\Psi}_{b} \mathbf{T}_{b}^{H}+\lambda \mathbf{I},
$$

where $\boldsymbol{\Psi}_{a}=2 \mathbf{A}^{2} \mathbf{C}_{\mathbf{e}_{\mathbf{h}}}+4 \mathbf{A}^{2} \hat{\mathbf{h}}^{2} \mathbf{C}_{\mathbf{e}_{\mathbf{h}}}, \boldsymbol{\Psi}_{b}=\mathbf{A}^{2} \mathbf{C}_{\mathbf{g}} \mathbf{C}_{\mathbf{e}_{\mathbf{h}}}, \lambda=n \sigma_{n}^{2}\left(\sum_{i=1}^{n} \alpha_{i}^{2} \sigma_{h i}^{2}+N\right)$.

From the equation (10), $\mathbf{s}_{a}$ can be used to compute the LMMSE estimator of $\mathbf{g}$ as

$$
\hat{\mathbf{g}}=\mathbf{C}_{\mathbf{g}}\left(\mathbf{T}_{b} \mathbf{A} \hat{\mathbf{h}}\right)^{H}\left[\left(\mathbf{T}_{b} \mathbf{A} \hat{\mathbf{h}}\right) \mathbf{C}_{\mathbf{g}}\left(\mathbf{T}_{b} \mathbf{A} \hat{\mathbf{h}}\right)^{H}+\mathbf{C}_{\mathbf{N}_{t} \mid \hat{\mathbf{h}}}\right]^{-1}\left(\mathbf{Y}_{a}^{s}-\mathbf{T}_{a} \mathbf{A} \hat{\mathbf{h}}^{2}\right)
$$

The corresponding $\mathbf{g}$ estimation error variance conditioned on $\hat{\mathbf{h}}$ can be obtained as 


$$
\mathbf{C}_{\Delta \mathbf{g} \hat{\mathbf{h}}}=\left(\mathbf{C}_{\mathbf{g}}^{-1}+\left(\mathbf{T}_{b} \mathbf{A} \hat{\mathbf{h}}\right)^{H} \mathbf{C}_{\mathbf{N}_{t} \hat{\mathbf{h}}}^{-1} \mathbf{T}_{b} \mathbf{A} \hat{\mathbf{h}}\right)^{-1}
$$

For the inverse matrix $\boldsymbol{\Theta}=\mathbf{I}+\mathbf{X B X} \mathbf{X}^{\mathbf{H}}$, there is

$$
\mathbf{\Theta}^{-1}=\mathbf{I}-\mathbf{X}\left(\mathbf{B}^{-1}+\mathbf{\Omega}\right)^{-1} \mathbf{X}^{\mathbf{H}}
$$

where $\boldsymbol{\Omega}=\mathbf{X}^{\mathbf{H}} \mathbf{X}$.

The $\mathbf{C}_{\mathbf{N}_{t} \mid \hat{\mathbf{h}}}$ presented in (11) can be worked out by the similar derivation to the equation (14), where $\mathbf{X}=\left[\mathbf{T}_{a}, \mathbf{T}_{b}\right], \mathbf{B}=\frac{1}{\lambda}\left[\begin{array}{ll}\boldsymbol{\Psi}_{a} & \\ & \boldsymbol{\Psi}_{b}\end{array}\right], \mathbf{C}_{\mathbf{N}_{t} \hat{\mathbf{h}}}=\lambda\left(\mathbf{I}+\mathbf{X B X} \mathbf{X}^{\mathbf{H}}\right)$. Then we can obtain

$$
\mathbf{C}_{\mathbf{N}_{t} \mid \hat{\mathbf{h}}}^{-1}=\frac{1}{\lambda}\left(\mathbf{I}-\mathbf{X}\left(\mathbf{B}^{-1}+\mathbf{\Omega}\right)^{-1} \mathbf{X}^{\mathbf{H}}\right)
$$

So (13) can be rewritten as

$$
\mathbf{C}_{\Delta \mathbf{g} \hat{\mathbf{h}}}=\left(\mathbf{C}_{\mathbf{g}}^{-1}+\left(\mathbf{T}_{b} \mathbf{A} \hat{\mathbf{h}}\right)^{H} \frac{1}{\lambda}\left(\mathbf{I}-\mathbf{X}\left(\mathbf{B}^{-1}+\mathbf{\Omega}\right)^{-1} \mathbf{X}^{\mathbf{H}}\right) \mathbf{T}_{b} \mathbf{A} \hat{\mathbf{h}}\right)^{-1}
$$

And the MSE of $\mathbf{g}$ is

$$
\begin{aligned}
M S E & =E\left\{\operatorname{tr}\left(\mathbf{C}_{\Delta \mathbf{g} \mid \mathbf{h}}\right)\right\} \\
& =\sum_{i=1}^{n} \sigma_{g i}^{2} \underbrace{E\left\{\frac{\Gamma_{a i}+\Gamma_{b i}\left|\bar{h}_{i}\right|^{2}}{\Gamma_{a i}+\left|\overline{h_{i}}\right|^{2}}\right\}}_{\Sigma_{i}},
\end{aligned}
$$

where $\quad \Gamma_{a i}=\frac{\sigma_{n}^{2}}{\sigma_{h i}^{2} P_{r i}}+\frac{\sigma_{n}^{2}\left(\sigma_{n}^{2}+\sigma_{h i}^{2} P_{r i}\right)}{\alpha_{i}^{2}\left(\sigma_{n}^{2}+\sigma_{g i}^{2} P_{b i}\right) \sigma_{h i}^{4} P_{r i}} \quad, \quad \Gamma_{b i}=\frac{\sigma_{n}^{2}}{\sigma_{n}^{2}+\sigma_{g i}^{2} P_{b i}}$ $P_{r i}=\beta_{i} P_{t}-\alpha_{o p t \_}^{2}\left(\sigma_{h i}^{2} P_{a i}+\sigma_{g i}^{2} P_{b i}+N \sigma_{n}^{2}\right)$.

From (6), we know the MSE in (17) is an equation about $\beta_{i}$ and $\left|\overline{h_{i}}\right|^{2}=\hat{h}_{i} / \sigma_{\hat{h}_{i}} \sim C N(0,1)$. After some integral calculation, we can obtain the closed-form expression of $\Sigma_{i}$ as

$$
\Sigma_{i}=\Gamma_{b i}-\Gamma_{a i}\left(1-\Gamma_{b i}\right) \exp \left(\Gamma_{a i}\right) \operatorname{Ei}\left(-\Gamma_{a i}\right),
$$


where $\operatorname{Ei}(\mathrm{x})=\int_{-\infty}^{\mathrm{x}} \frac{e^{t}}{t} d t$. Applying (18) into (17), we obtain

$$
M S E=\sum_{i=1}^{n}\left\{\sigma_{g i}^{2} \Gamma_{b i}-\sigma_{g i}^{2} \Gamma_{a i}\left(1-\Gamma_{b i}\right) \exp \left(\Gamma_{a i}\right) \operatorname{Ei}\left(-\Gamma_{a i}\right)\right\}
$$

Then, we can optimize the power allocation factor $\beta_{i}$ through minimizing MSE.

$$
\begin{aligned}
& \min _{\beta_{i}} \operatorname{MSE} \\
& \text { s.t. } \sum_{i=1}^{n} \beta_{i}=1,0 \leq \beta_{i} \leq 1, i=1,2, \ldots, n
\end{aligned}
$$

From (19), it is obvious that we can minimize the MSE by minimizing the $\Gamma_{a i}$. Through the derivation of $\Gamma_{a i}$ and after some tedious mathematical operations, we could see that $\Gamma_{a i}$ is a convex function. Overall, based on (20), we can obtain the optimal power allocation factor $\beta_{\text {opt } \_i}$ by calculating this optimization problem, which can be easily computed by using the "fmincon" in MATLAB.

\section{Simulation}

In this section, numerical simulations are provided to test the performance of optimal power allocation for MPS AF based TWRN. The parameters are set as following: $\sigma_{h i}^{2}=\sigma_{g i}^{2}=1, \sigma_{n}^{2}=1, N=8$, and we assume that $s_{a i}$ and $s_{b i}$ are symmetrical, the SNR of $s_{a i}$ and $s_{b i}$ are defined as $S N R_{i}=\frac{P_{a i}}{\sigma_{n}^{2}}=\frac{P_{b i}}{\sigma_{n}^{2}}$, and the SNR of $R$ is given as $S N R_{t}=\frac{P_{t}}{\sigma_{n}^{2}}$.

We compared three methods as shown in following figures, where the proposed method is denoted as OPA with $\alpha_{i}=\alpha_{o p t_{-} i}$ and $\beta_{i}=\beta_{o p t_{-} i}$, the conventional method, in which $\alpha_{i}=0.5 \sqrt{\frac{\beta_{i} P_{t}}{\sigma_{h i}^{2} P_{a i}+\sigma_{g i}^{2} P_{b i}+N \sigma_{n}^{2}}}$ and $\beta_{i}=\frac{1}{n}$, is marked by EPA, and the power allocation algorithm mentioned in [6], where $\alpha_{i}=\alpha_{\text {opt }{ }_{-} i}$ and $\beta_{i}=\frac{1}{n}$, is marked by $\mathrm{PA}_{[6]}$. The reader is encouraged to find more performance comparisons with other training scheme in our previous work $[4,6]$. We also assume that $S N R_{1}=S N R_{2}=0 d B$ and $S N R_{3}$ varies from $-10 \mathrm{~dB}$ to $10 \mathrm{~dB}$.

Fig. 2 gives the simulation results when $S N R_{t}=10 d B$, which is defined as a low SNR scenario. It shows that the system MSE decreases when $S N R_{3}$ increases, and the OPA outperforms the others obviously. Fig. 3 shows the optimal power allocation factors versus $S N R_{3}$ when $S N R_{t}=10 \mathrm{~dB}$. Since we set $S N R_{1}=S N R_{2}=0 d B$, the factors $\beta_{1}$ is always equal 
to $\beta_{2}$ and $\beta_{1}=\beta_{2}=\beta_{3}=0.3333$ when $S N R_{3}=0 d B$. From Fig. 3, we can see that $\beta_{1}=\beta_{2}>\beta_{3}$ when $S N R_{3}<0 d B$, the lower $S N R_{3}$ the smaller $\beta_{3}$, and $\beta_{3}$ is near to zero when $S N R_{3}$ is lower than a threshold, such as $-2 \mathrm{~dB}$.

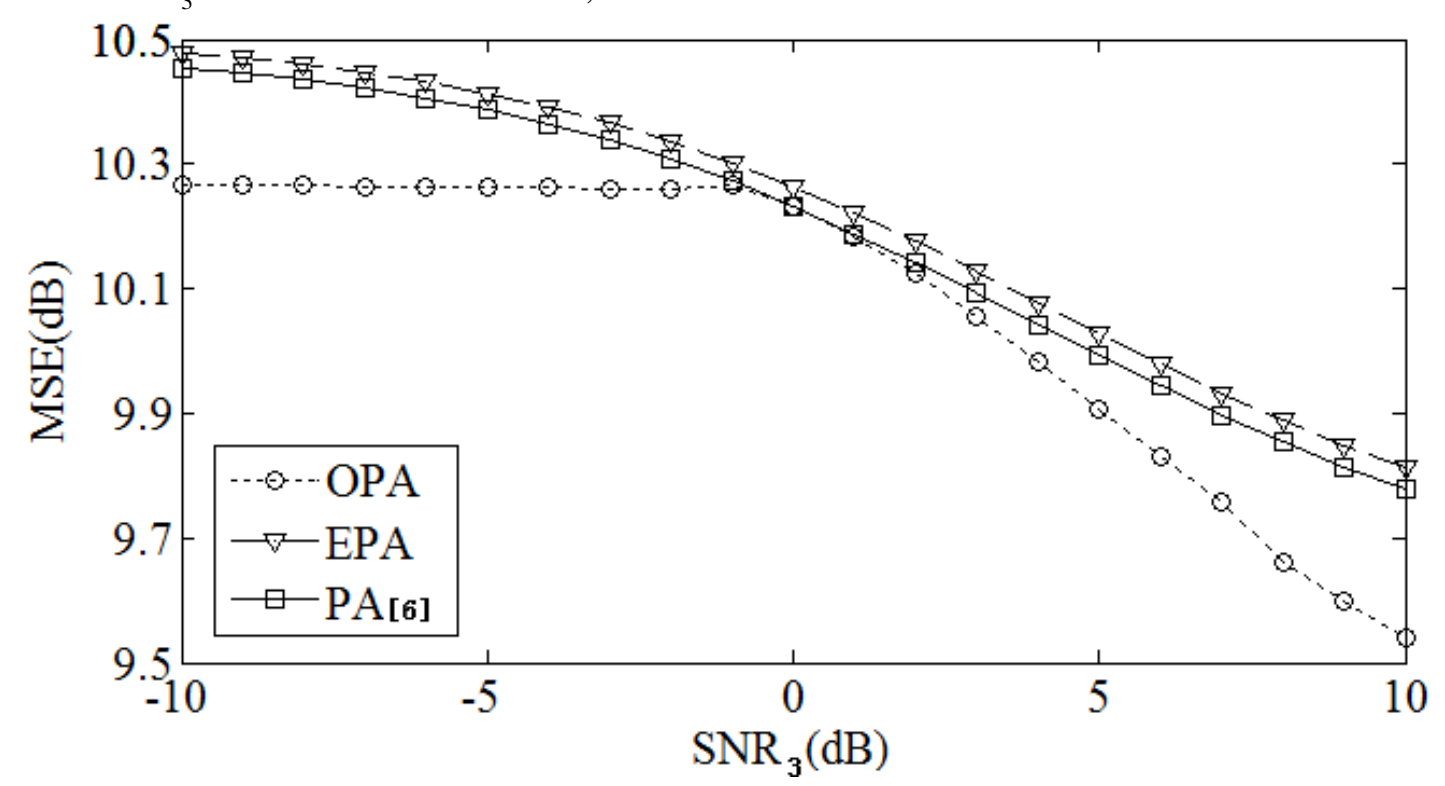

Fig. 2. MSE versus $S N R_{3}$ under OPA, EPA and $\mathrm{PA}_{[6]}$ when $S N R_{t}=10 \mathrm{~dB}$ and $n=3$

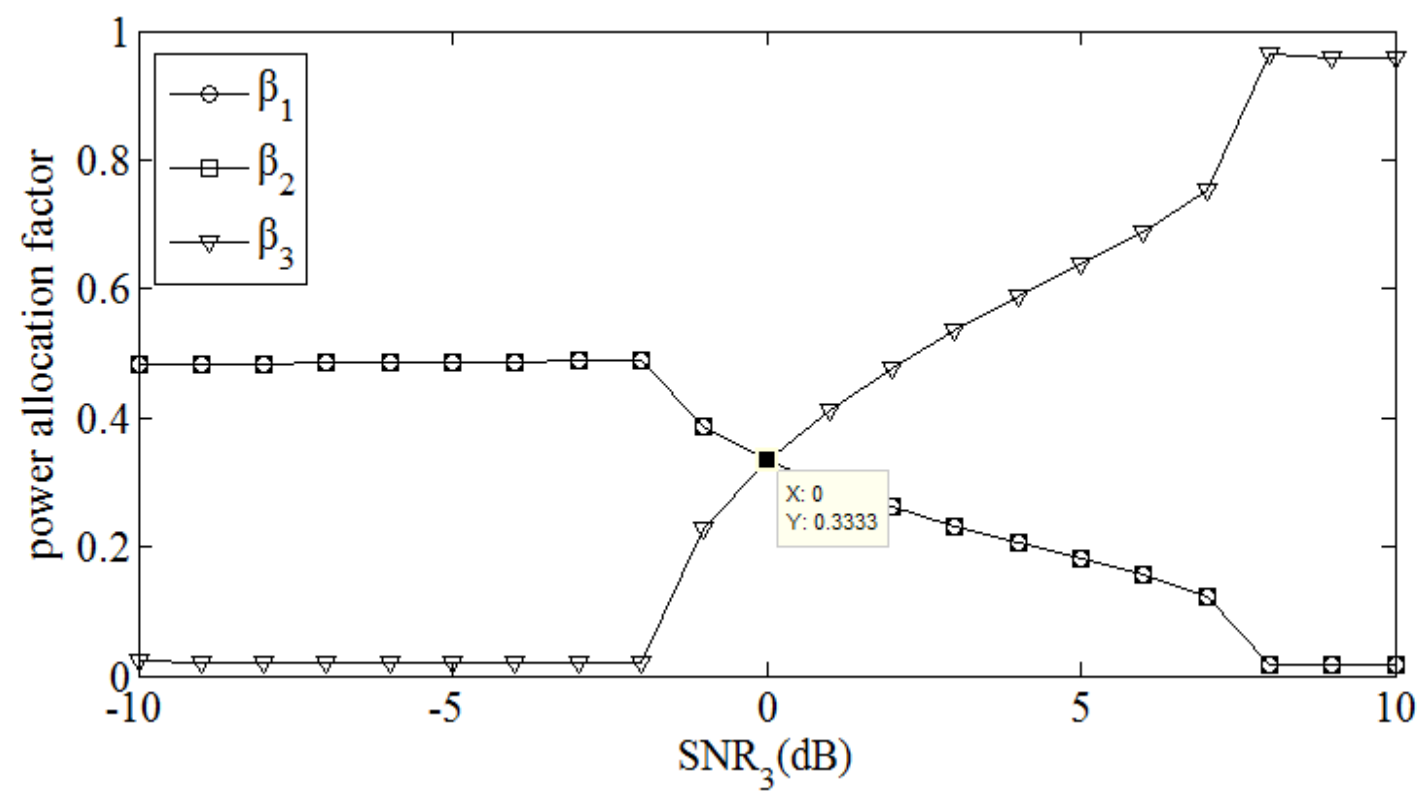

Fig. 3. Power allocation factor $\beta_{1}, \beta_{2}$ and $\beta_{3}$ versus $S N R_{3}$ when $S N R_{t}=10 d B$ and $n=3$

Fig. 4 presents the simulation results when relay $S N R_{t}=15 d B$, which is defined as a middle SNR scenario. Similar to what are shown in Fig. 2, the system MSE decreases when $S N R_{3}$ increases. We can see the OPA outperforms EPA and $\mathrm{PA}_{[6]}$ obviously when $S N R_{3}$ is 
low, which is explained in Fig. 5. Fig. 5 shows the optimal power allocation factors versus $\mathrm{SNR}_{3}$ when $S N R_{t}=15 d B$. Since $S N R_{1}=S N R_{2}=0 d B$, we get the factor $\beta_{1}=\beta_{2}$, as well as $\beta_{1}=\beta_{2}=\beta_{3}=0.3333$ when $S N R_{3}=0 d B$. From Fig. 5, we can see that $\beta_{1}=\beta_{2}>\beta_{3}$ when $S N R_{3}<0 d B$, the lower $S N R_{3}$ the smaller $\beta_{3}$, and $\beta_{3}$ is near to zero when $S N R_{3}$ is lower than a threshold, such as $-7 \mathrm{~dB}$.

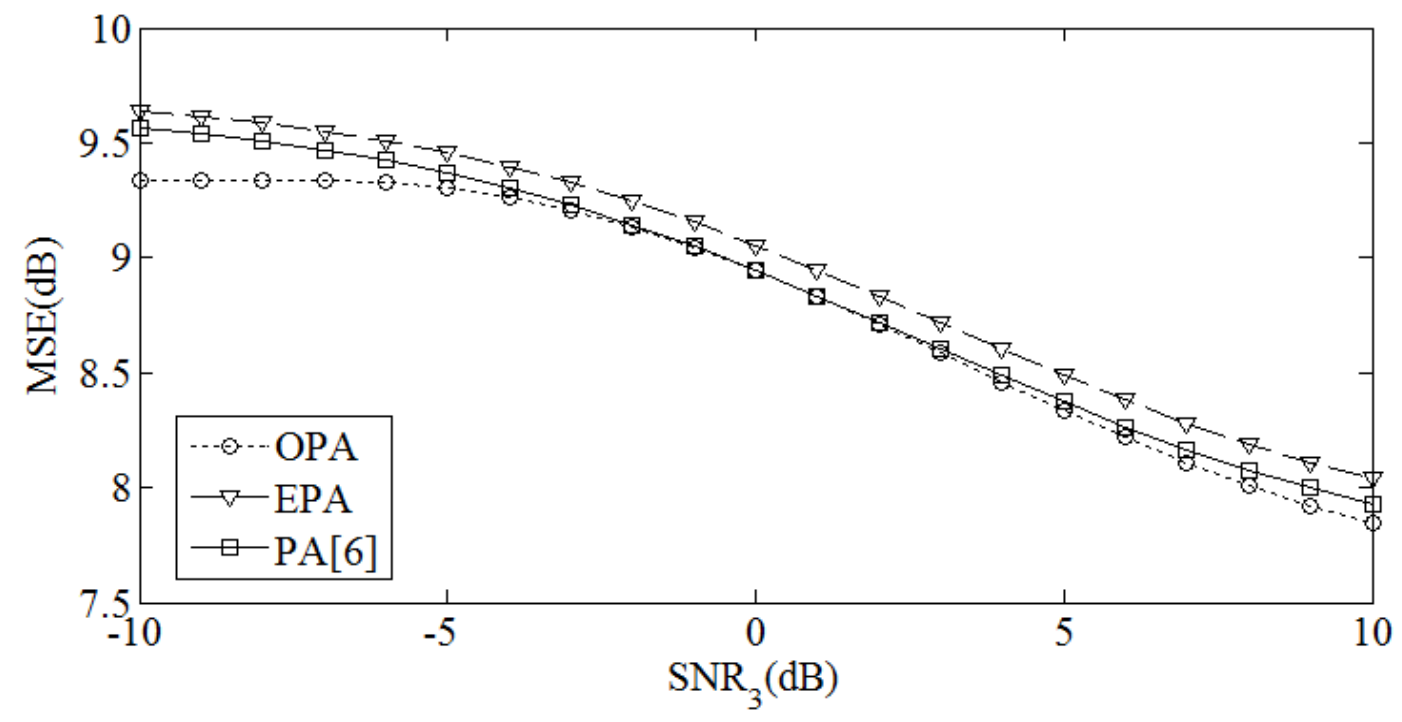

Fig. 4. MSE versus $S N R_{3}$ under OPA, EPA and $\mathrm{PA}_{[6]}$ when $S N R_{t}=15 d B$ and $n=3$

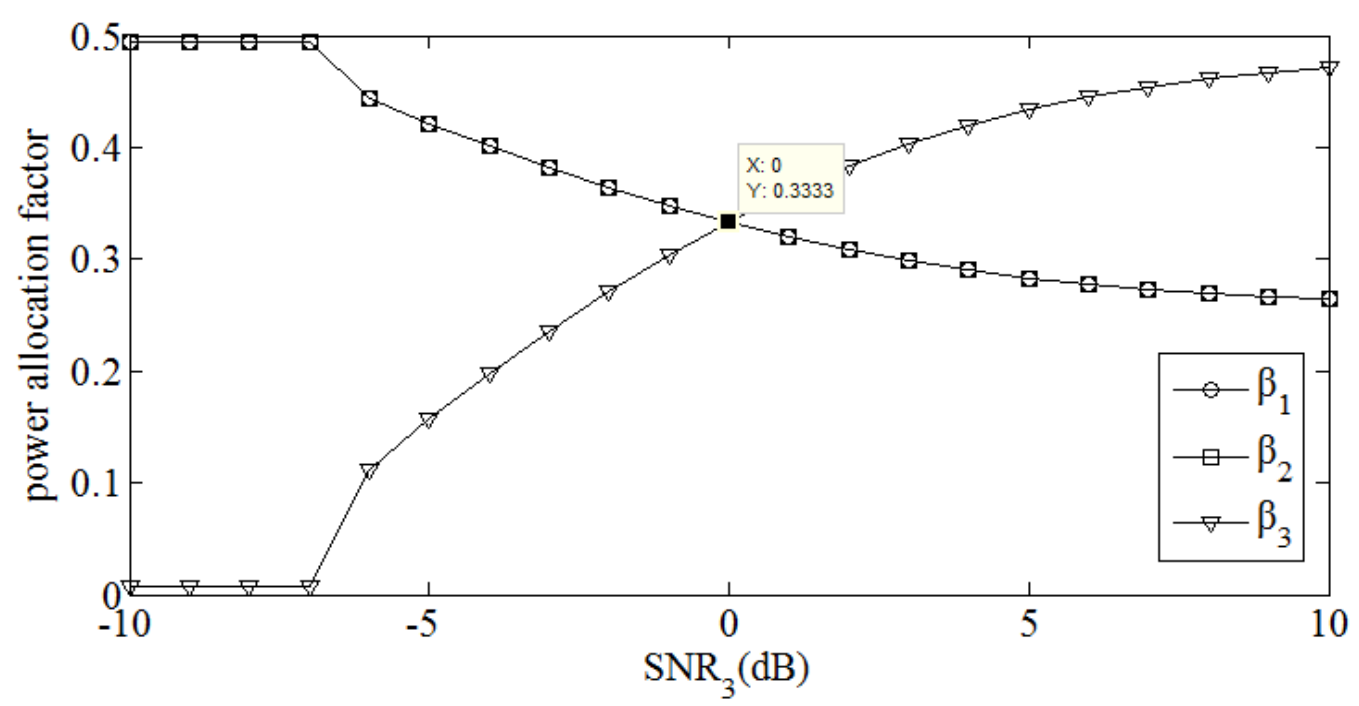

Fig. 5. Power allocation factor $\beta_{1}, \beta_{2}$ and $\beta_{3}$ versus $S N R_{3}$ when $S N R_{t}=15 d B$ and $n=3$

Fig. 6 and Fig. 7 give the performance when $S N R_{t}$ is high. Here, the relay SNR is $20 \mathrm{~dB}$. The performance is similar to the results in Fig. 2 and Fig. 3, except for that the curves are more smooth in Fig. 7. This is because when the SNR of training data is above a level, the MSE changes little with the increasing of training data's power, which means more power needs to be allocated to the source pairs with small SNR. 


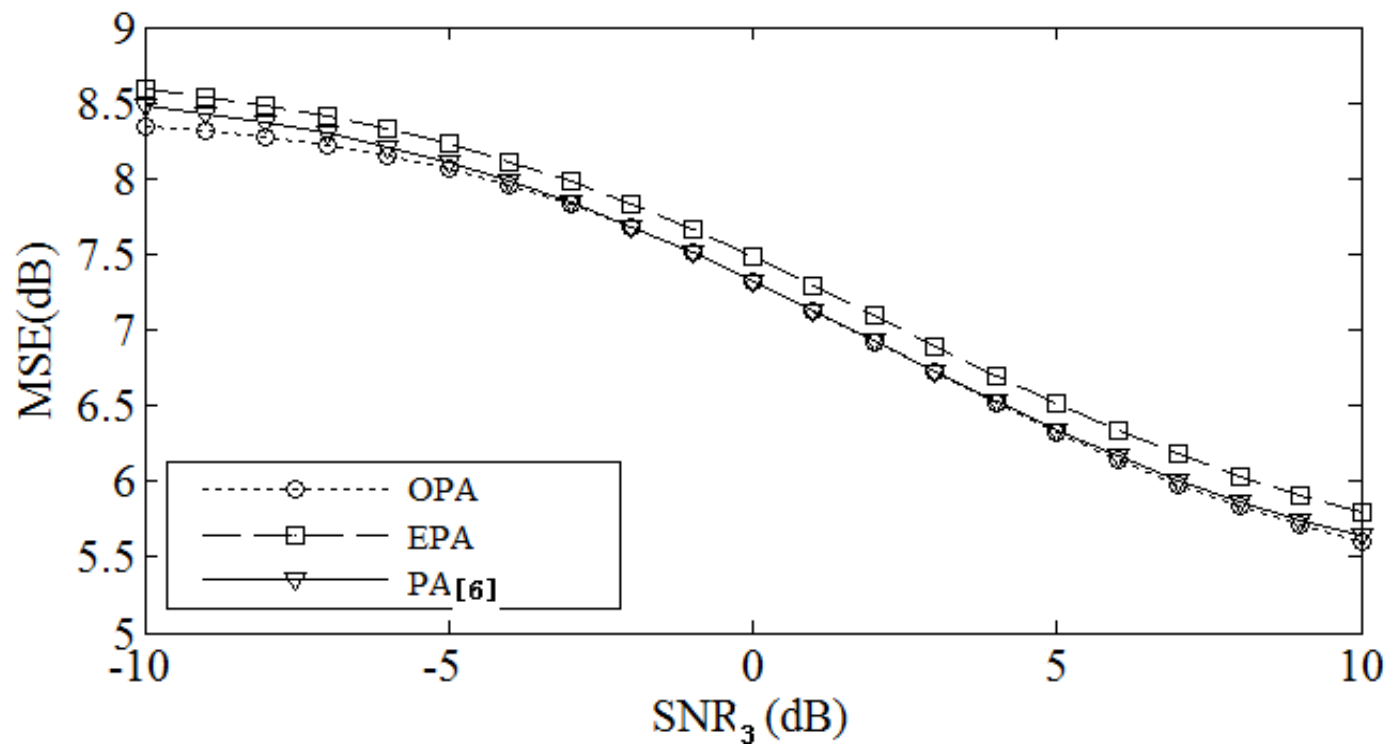

Fig. 6. MSE versus $S N R_{3}$ under OPA, EPA and $\mathrm{PA}_{[6]}$ when $S N R_{t}=20 \mathrm{~dB}$ and $n=3$

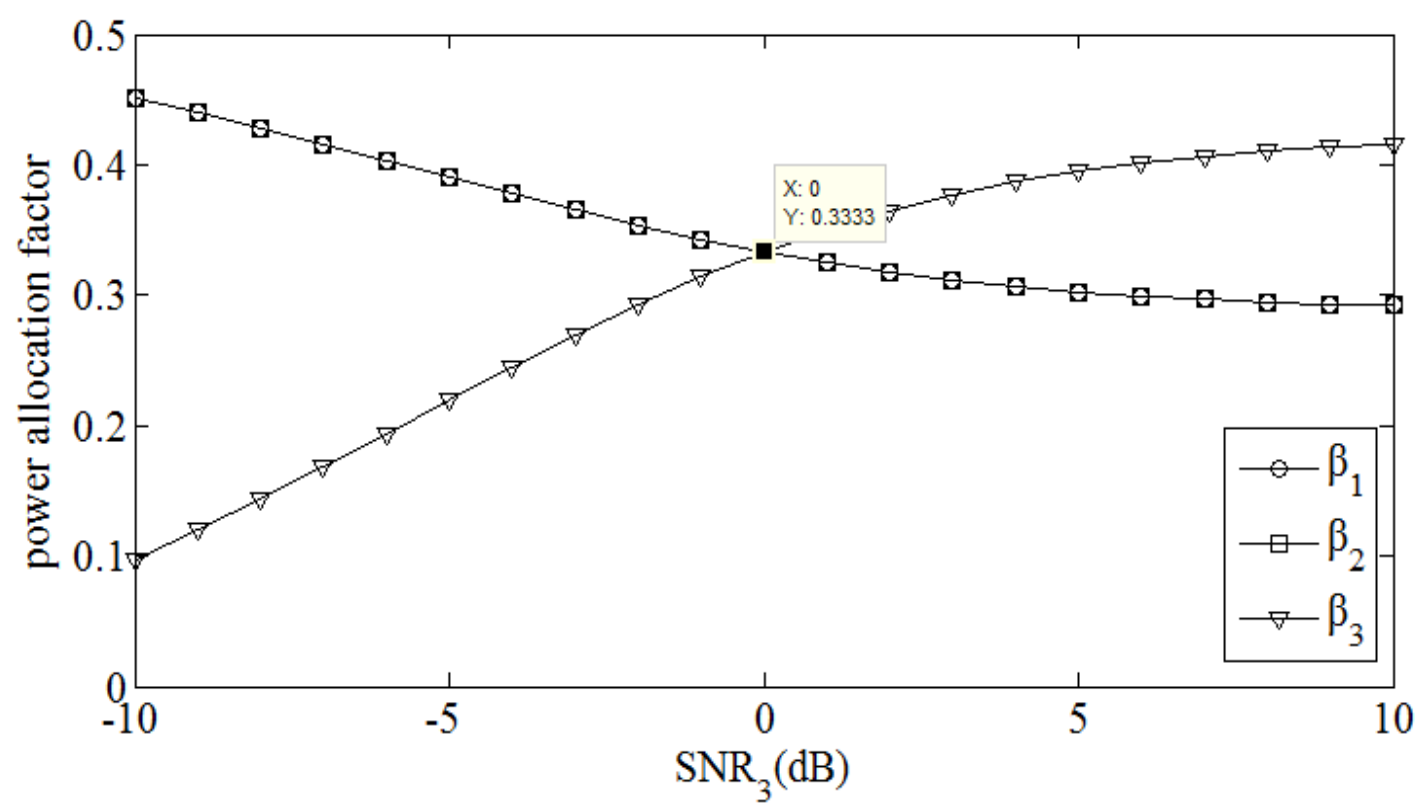

Fig. 7. Power allocation factor $\beta_{1}, \beta_{2}$ and $\beta_{3}$ versus $S N R_{3}$ when $S N R_{t}=20 d B$ and $n=3$

In order to quantize the performance improvement between the OPA and the other existing algorithms, we define the gain of $M S E_{\text {equ }}$ and $M S E_{[6]}$ as $M S E_{\text {equ }}$ gain $=\frac{M S E_{\text {equ }}}{M S E_{\text {opt }}}$ and $M S E_{[6]}$ gain $=\frac{M S E_{[6]}}{M S E_{o p t}}$, respectively, which are shown in Fig. 8 and Fig. 9. In these figures, 
we set the relay $S N R$ as $S N R_{t}=20 d B, S N R_{i}$ s are equal when $\mathrm{i}=1,3,4$. Fig. 8 shows the MSE gain versus $S N R_{2}$, where the source pair number $n=3$. In Fig. 9, three different source pair numbers $n=2,3$ and 4 are adopted. Generally speaking, the larger differences between $S N R_{i}$ and $S N R_{2}$, the higher gain we get. Furthermore, when $S N R_{2}$ is large, the MSE gain becomes bigger with the number of source pair increases, but the computation complexity increases dramatically. When $S N R_{2}$ is small, the MSE gain becomes smaller with the source number increases. These phenomena give a reference to choose the acceptable source-pair number when designing a real system.

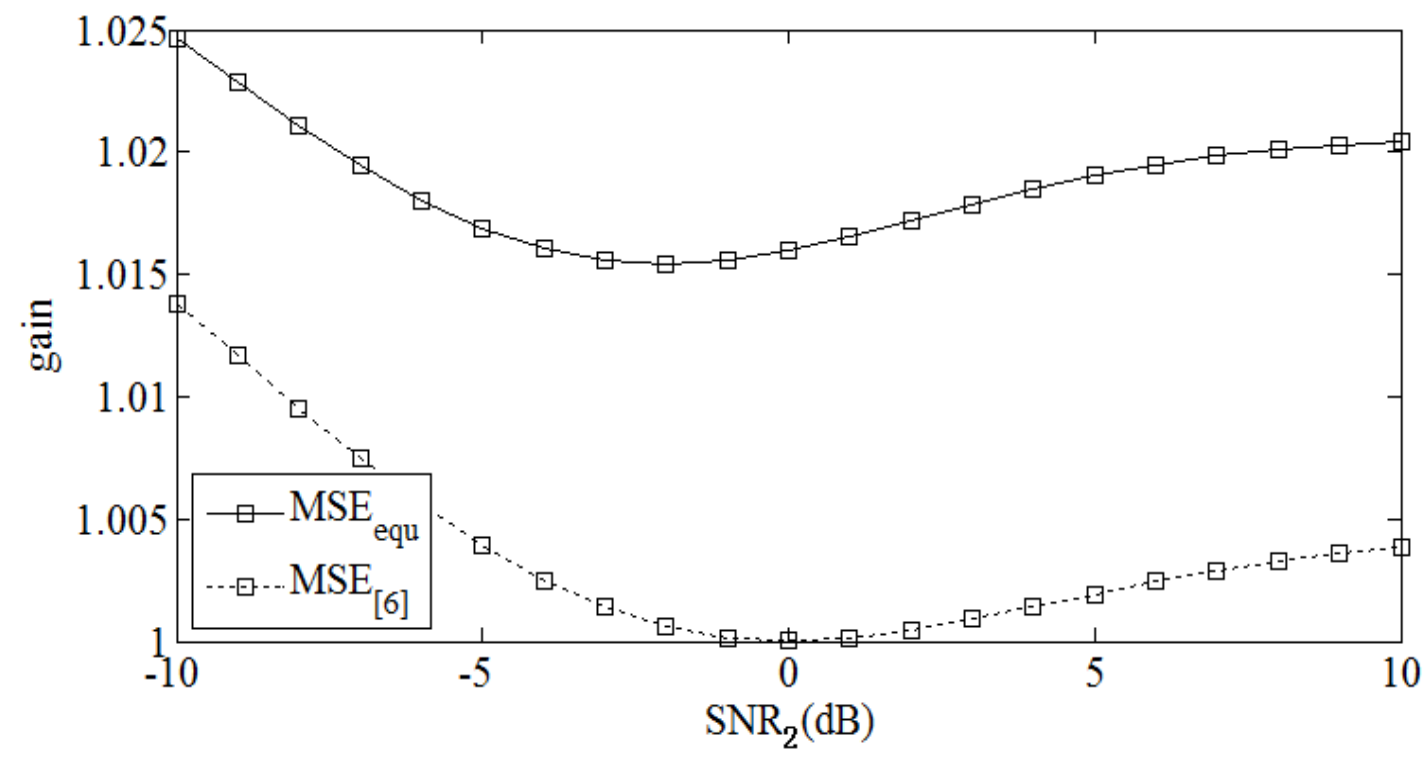

Fig. 8. MSE gain versus $S N R_{2}$ when $S N R_{t}=20 d B$

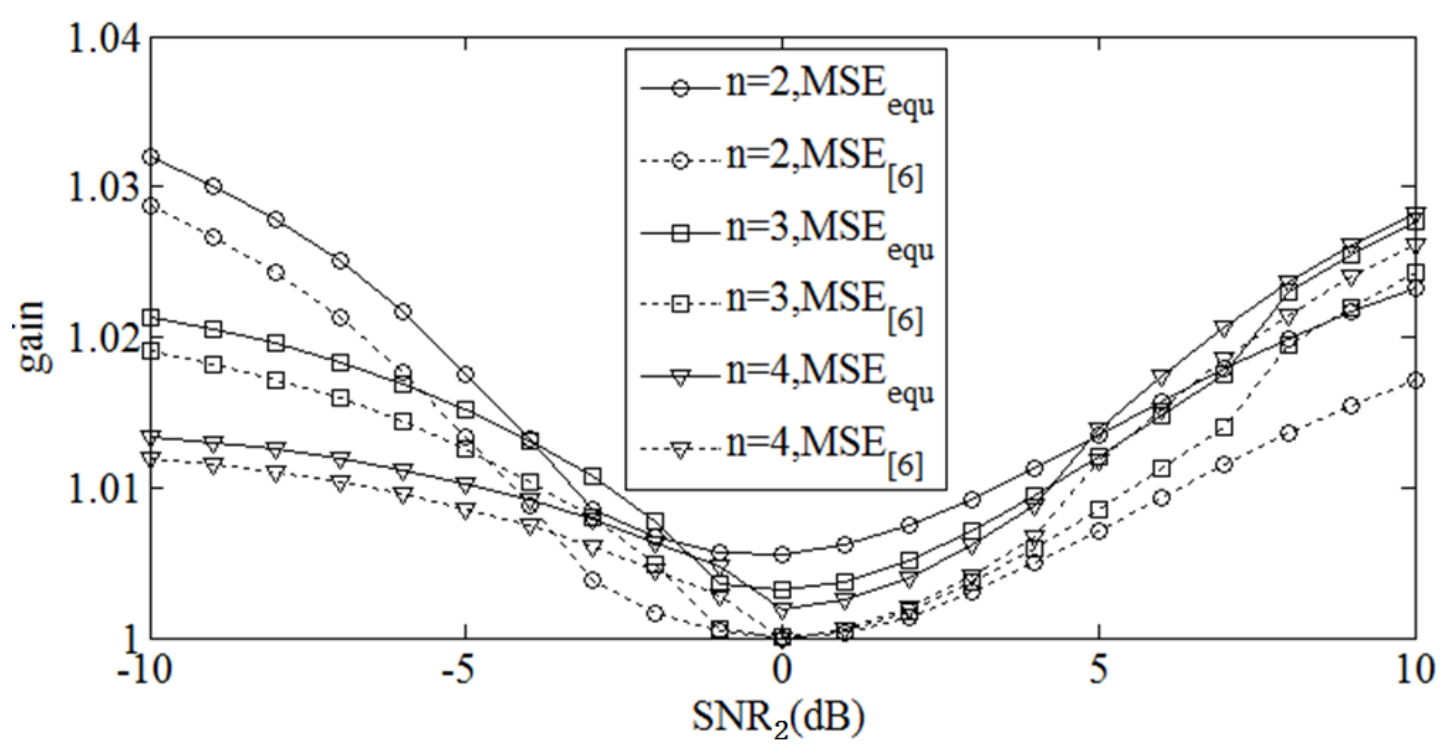

Fig. 9. MSE gain versus $S N R_{2}$ when $S N R_{t}=20 d B$ 


\section{Conclusion}

In this paper, we introduce a scheme to estimate channel state information for a typical $2 n+1$-node TWRN, and propose a new algorithm to minimize the MSE by computing the optimal power allocation factors at relay node for different sources. The proposed method has gained better performance especially when the relay power is low. The numerical results give a reference to choose the acceptable source-pair number when designing a real system.

\section{References}

[1] Feifei Gao, Tao Cui and Arumugam Nallanathan, "On channel estimation and optimal training design for amplify and forward relay networks," IEEE Transactions on Wireless Communications, vol. 7, no. 5, pp. 1907-1916, 2008. Article (CrossRef Link).

[2] Aris S. Lalos, Athanasios A. Rontogiannis and Kostas Berberidis, "Frequency Domain Channel Estimation for Cooperative Communication Networks," IEEE Transactions on Signal Processing, vol. 58, no. 6, pp. 3400-3405, 2010. Article (CrossRef Link).

[3] Ma Jun, et al, "Pilot Matrix Design for Estimating Cascaded Channels in Two-Hop MIMO Amplify-and-Forward Relay Systems," IEEE Transactions on Wireless Communications, vol. 10, no. 6, pp. 1956-1965, 2011. Article (CrossRef Link).

[4] Zhang Shun, et al, "Segment Training Based Individual Channel Estimation in One-Way Relay Network with Power Allocation,” IEEE Transactions on Wireless Communications, vol. 12, no. 3, pp. 1300-1309, 2013. Article (CrossRef Link).

[5] Xing Chengwen, et al, "A General Robust Linear Transceiver Design for Multi-Hop Amplify-and-Forward MIMO Relaying Systems," IEEE Transactions on Signal Processing, vol. 61, no. 5, pp. 1196-1209, 2011. Article (CrossRef Link).

[6] Zhang Shun, et al, "Study of Segment Training Based Individual Channel Estimation for Two-Way Relay Network,” IEEE Communications Letters, vol. 17, no. 2, pp. 401-404, 2013. Article (CrossRef Link).

[7] Godfrey Okeke, Witold A. Krzymien and Yindi Jing, "Beamforming in Non-Regenerative MIMO Broadcast Relay Networks," IEEE Transactions on Signal Processing, vol. 60, no. 12, pp. 6641-6654, 2012. Article (CrossRef Link).

[8] Mohamed Fadel, Amr El-Keyi and Ahmed Sultan, "QOS-Constrained Multiuser Peer-to-Peer Amplify-and-Forward Relay Beamforming,” IEEE Transactions on Signal Processing, vol. 60, no. 60, pp. 1397-1408, 2012. Article (CrossRef Link).

[9] S. Yiu and R. Schober; L. Lampe, "Distributed space-time block coding," IEEE Transactions on Communications, vol. 54, no. 7, pp. 1195-1206, 2006. Article (CrossRef Link).

[10] Jing Yindi and B. Hassibi, "Distributed Space-Time Coding in Wireless Relay Networks," IEEE Transactions on Wireless Communications, vol. 5, no. 12, pp. 3524-3536, 2006. Article (CrossRef Link).

[11] Ashkan Kalantari, Ehsan Soleimani-Nasab and Mehrdad Ardebilipour, "Performance analysis of multi-antenna relay networks with imperfect channel estimation," in Proc. of 20th Telecommunications Forum (TELFOR), pp. 823-826, Nov. 2012. Article (CrossRef Link).

[12] Ting Kong and Yingbo Hua, "Optimal Design of Source and Relay Pilots for MIMO Relay Channel Estimation,” IEEE Transactions on Signal Processing, vol. 59, no. 9, pp. 4438-4446, 2011. Article (CrossRef Link).

[13] F. Gao, R. Zhang and Y. -C. Liang, "Optimal channel estimation and training design for two-way relay networks,” IEEE Transactions on Communications, vol. 57, no. 10, pp. 3024-3033, 2009. Article (CrossRef Link).

[14] Bin Jiang, Feifei Gao, et al, "Channel estimation and training design for two-way relay networks with power allocation," IEEE Transactions on Wireless Communications, vol. 9, no. 6, pp. 2022-2032, 2010. Article (CrossRef Link).

[15] J. M. Kang and H. M. Kim, “Training Designs for Estimation of Spatially Correlated Fading 
Channels in MIMO Amplify-and-Forward Two-Way Multi-Relay Networks,” IEEE Communications Letters, vol. 20 no. 4, pp. 772-775, 2016. Article (CrossRef Link).

[16] Choo W. R. Chiong, Yue Rong and Yong Xiang, "Channel Training Algorithms for Two-Way MIMO Relay Systems,” IEEE Transactions on Signal Processing, vol. 61, no. 16, pp. 3988-3998, 2013. Article (CrossRef Link).

[17] M. Y1ldırım and H. Ilhan, “OFDM based link adaptive one-way DF relaying,” AEU- International Journal of Electronics and Communications, vol. 70, no. 5, pp. 657-661, 2016. Article (CrossRef Link).

[18] E. Yilmaz, et al, "Multi-Pair Two-Way Relay Channel with Multiple Antenna Relay Station," International Communications Conference 2010, pp. 1-5, May, 2010. Article (CrossRef Link).

[19] Yongyu Dai and Xiaodai Dong, "Power allocation for multi-pair massive MIMO two-way AF relaying with linear processing,” IEEE Trans Wireless Commun 2016, vol. 15, no.9, pp. 5932-5946, 2016. Article (CrossRef Link).


Xiandeng He received the B.S. degree in 2004 and Ph. D. degree in 2010 in communication and signal processing from Xidian university, Xi'an, China. Since August 2010, he has joined the State Key Laboratory of Integrated Services Networks, Xidian University. And now he is an associate Professor in the School of Telecommunication and Engineering, Xidian University. His main research topics focus on radio communication, anti-jamming communication, synchronization techniques, channel estimation, and Ad-hoc network.

Ronghua Zhou received the M.S. degree from Hebei University of Economics and Business, Hebei, China, in 2013. She is currently pursuing the B.S. degree in communication and signal processing with the State Key Laboratory of Integrated Services Networks, Xidian University, Xi'an, China. Her research interests are channel estimation, relay networks, signal detection and cooperation communication.

Nan Chen is a professor, senior member of China Institute of Electronic, Director of the department of electronic technology and deputy director of electronic and electronic teaching base in Xidian University. He received the M.S. degree in 1987 and B.S. degree in 1993 in the School of Communication and Engineering, Xidian university, Xi'an, China. He has been awarded the first and second prize of national teaching achievement. His main research topics focus on wireless communication, anti-jamming communication, and channel estimation.

Shun Zhang received the B.S. degree in 2007 in communication engineering from Shangdong University, Jinan, China, received the Ph. D. degree in 2014 in communication and signal processing from Xidian university, Xi'an, China. Since August 2014, he has joined the State Key Laboratory of Integrated Services Networks, Xidian University and now is an associate Professor in the School of Telecommunication and Engineering, Xidian University. His research interests include MIMO OFDM systems, relay networks, and detection and parameter estimation theory. 